\title{
RESIDUOS DE CELULARES Y TABLETAS: INCIDENCIA DEL NIVEL DE ESCOLARIDAD EN LAS ACTITUDES Y PERCEPCIONES FRENTE A SU MANEJO
}

\section{RESIDUES OF CELL PHONES AND TABLETS: INCIDENCE OF THE SCHOOL LEVEL IN THE ATTITUDES AND PERCEPTIONS IN FRONT OF ITS HANDLING}

\begin{abstract}
Yois Pascuas R. ${ }^{1}$, Diana Chico V. ${ }^{2}$, Sergio Hernández R. ${ }^{3}$
${ }^{1}$ Candidata a Doctora en Educación y Cultura Ambiental, Magíster en Ciencias de la Información y las Comunicaciones, Ingeniera de Sistemas, Docente Facultad de Ingeniería. Universidad de la Amazonia, Calle 17 Diagonal 17 con Carrera 3F Barrio Porvenir, Florencia-Caquetá, Colombia, e-mail: y.pascuas@udla.edu.co; ²Estudiante de Maestría en Tecnologías de la Información y las Comunicaciones, Ingeniera de Sistemas, Docente Facultad de Ingeniería. Universidad de la Amazonia, Calle 17 Diagonal 17 con Carrera 3F Barrio Porvenir, Florencia-Caquetá, Colombia, e-mail: d.chico@udla.edu.co; ${ }^{3}$ Magíster en Ciencias de la Información y las Comunicaciones, Especialista en Matemáticas Avanzadas, Licenciado en Matemáticas y Física, Docente Facultad de Ciencias de la Educación. Universidad de la Amazonia, Calle 17 Diagonal 17 con Carrera 3F Barrio Porvenir, Florencia-Caquetá, Colombia, e-mail: sehernandez@uniamazonia.edu.co
\end{abstract}

Rev. U.D.C.A Act. \& Div. Cient. 21(1): 243-252, Enero-Junio, 2018

https://doi.org/10.31910/rudca.v21.n1.2018.683

RESUMEN

El avance tecnológico ha traído consigo la generación de residuos de celulares y tabletas. Esta tendencia seguirá en aumento, dado el fácil acceso a estos dispositivos. En el siguiente estudio, se relaciona la incidencia del nivel de escolaridad, sobre las actitudes y las percepciones, que se tienen sobre el manejo de los residuos provenientes de celulares y de tabletas, con el fin de ahondar en esta problemática y aportar insumos para la creación de estrategias y de políticas encaminadas al desarrollo sustentable. Para esto, se aplicó una encuesta a 450 personas que habitan en la ciudad de Florencia - Caquetá, Colombia. Se realizó un test de independencia, que permitió decidir si el nivel de escolaridad incide sobre las actitudes y las percepciones. Los resultados obtenidos indicaron que no existe tal dependencia con las actitudes, pero sí con las percepciones, lo que demuestra la inconsistencia del ser humano entre lo que piensa y cómo actúa. Un hallazgo relevante en el presente estudio es que, en su gran mayoría, estos dispositivos o sus partes, después de un tiempo, terminan en la basura, junto con los residuos convencionales.

Palabras clave: Ambiental, comportamiento, dispositivo, electrónica, RAEE.

\section{SUMMARY}

The technological advance has brought with it the generation of cell and tablet waste. This trend will continue to increase, given the easy access to these devices. In the following study, the incidence of schooling level is related to attitudes and perceptions about the handling of cell and tablet waste, in order to deepen this problem and provide inputs for the creation of strategies and policies aimed at sustainable development. For this, a survey was applied to 450 people living in the city of Florencia Caquetá Colombia. An independence test was carried out, which allowed to decide if the level of schooling affects attitudes and perceptions. The results obtained indicated that there is no such dependence on attitudes, but with perceptions, this shows the human being's lack of balance between what he thinks and how he acts. A relevant finding in the present study, is that the vast majority of these devices or their parts, after a while end up in the trash, along with conventional waste.

Key words: Environmental, behavior, device, electronics, WEEE.

\section{INTRODUCCIÓN}

Los celulares y las tabletas son conocidos como aparatos electrónicos (Jaiswal et al. 2015) y cuando dejan de funcionar o no son útiles para el usuario, se consideran residuos de aparatos eléctricos y electrónicos (RAEE). Estos residuos, 
se están incrementando, debido a la oferta y la demanda de productos y servicios que prestan las tecnologías de la información y las comunicaciones (Özkır et al. 2015) y, en particular, a la tasa de obsolescencia, que es el tiempo de vida útil del aparato y que oscila entre 3 y 5 años (Román, 2014).

Esta tendencia, que va en aumento, amenaza la conservación de los ecosistemas y principalmente la salud pública, ya que incluyen elementos nocivos y altamente contaminantes (Song \& Li, 2014), como el plomo, el cromo, el mercurio, el cadmio y el arsénico (Jaiswal et al. 2015; Abdollahi et al. 2015), los cuales, podrían ser liberados y transportados a los cuerpos de agua, por medio de los lixiviados. Es así, como Cucchiella et al. (2015) consideran los RAEE, como la corriente de residuos de más rápido aumento en el mundo, con una tasa de crecimiento estimada del 3 al 5\% por año y donde menos del 10\% es reciclado (Hanselman \& Pegah, 2007).

Para tratar de darle solución al problema, los países desarrollados transportan los RAEE a países subdesarrollados, entre los cuales, se encuentran Ghana, Nigeria, Costa de Marfil, India y China, trasladando el problema de un lado a otro. Para impedir la ocurrencia de este fenómeno, se han realizado ingentes esfuerzos, con el propósito de controlar el movimiento transfronterizo de los desechos peligrosos, pero estas acciones, no han logrado los resultados esperados (Milovantseva \& Fitzpatrick, 2015; Robinson, 2009), por tal motivo, se requieren normas y políticas, sobre sistemas de gestión de residuos electrónicos, para evitar que los materiales tóxicos, contenidos en estos dispositivos, terminen en los rellenos sanitarios, zonas verdes y lugares públicos ( $\mathrm{Pa}$ nambunan-Ferse \& Breiter, 2013; Ping, 2009). Igualmente, se hace necesario brindar una adecuada educación (Orlins \& Guan, 2016) a los individuos, para que tomen conciencia del riesgo potencial de los RAEE, es decir, concientizar sobre los beneficios que trae el reciclaje de estos dispositivos (Perkins et al. 2014; Milovantseva \& Fitzpatrick, 2015; Panambunan-Ferse \& Breiter, 2013; Buisson \& Naidoo, 2014).

Como uno de los mecanismos para contrarrestar esta problemática, se han implementado actividades ecológicas relacionadas con los RAEE: parques ecológicos, redes de diseño verde, ecoetiquetas y proyectos de reciclaje (Chen et al. 2005); por ejemplo, Hanselman \& Pegah (2007) plantean que las instituciones de educación superior deben liderar las propuestas sobre el manejo de estos dispositivos, formas de prever los riesgos acarreados por los RAEE, mediante reutilización y reglamentación de estas actividades. Adicionalmente, se propone el uso de las TIC, como estrategia de implementación de cursos, para desarrollar tanto la conciencia y la responsabilidad ambiental (Daskolia \& Lambropoulos, 2009; Zhang et al. 2009; Roy et al. 2012), como también la aplicación de normas subjetivas de informática verde (Buisson \& Naidoo, 2014). Además, hay que mencionar que, actualmente, las redes sociales influyen en la toma de decisiones sobre el consumo (Pan \& Thomas, 2012), sin tener en cuenta los impactos ambientales que esto causa, dada la poca información disponible acerca de esta temática y la escasa legislación al respecto; sin embargo, en países como Colombia, que ha sido pionero en Latinoamérica en legislar sobre los RAEE, se han implementado políticas de gestión de RAEE. Es así como, en el 2013, se establece la Ley 1672, sobre la gestión integral; en el 2014, crea el Comité Nacional de RAEE, que formuló el plan de acción hasta el 2032; en el 2017, se lanzó la Política Nacional de Gestión Integral RAEE y en febrero de 2018, se adicionó al Decreto 1076 del 2015, aspectos sobre la gestión integral de RAEE, pero a pesar de estas apuestas, aún no se vislumbra incidencia directa, dado que las autoridades ambientales no la hacen cumplir (Ministerio de Ambiente y Desarrollo Sostenible, 2018).

Por otro lado, Fischer \& Haberl (2000) afirman que para satisfacer la alta demanda de este tipo de dispositivos electrónicos, se incrementan las labores de minería, con el propósito de obtener del subsuelo la materia prima para la fabricación de estos elementos, sobrecargando así la capacidad de los ecosistemas. Así, pues, sirve de ejemplo Colombia; el daño ambiental ocasionado da cuenta de 6.330 puntos dedicados a la extracción ilegal de oro, coltán y tungsteno (Torres, 2015), cerca de 200 mil hectáreas de ríos y de zonas selváticas deterioradas; zonas de páramo amenazadas por la búsqueda de oro en socavón; parques naturales nacionales (entre ellos, varios de la Amazonia), en la que hace presencia la minería criminal, cuya mano de obra de indígenas, de colonos y de extranjeros ilegales, se da a cambio de miseria. Situaciones estas, que son parte de la cadena orientada a la fabricación de componentes electrónicos, utilizados para optimizar procesos empresariales, el manejo de la información y las comunicaciones (Especiales Semana, 2016).

Según Rokeach (1968), las actitudes, se consideran como la organización de varias creencias centradas en un objeto específico, como, por ejemplo, físico o social, concreto o abstracto o una situación, lo que predispone a responder, de cierta manera, a través de una acción. Por otro lado, y en esta misma línea de argumentación, la percepción, ligada al pensamiento, se retoma como el proceso, por el cual, los individuos interpretan y organizan una sensación para producir una experiencia significativa del mundo. Es así, como percepción y actitud, son conceptos relacionados, aunque cada uno se enfoca en diferentes aspectos de las personas (Pickens, 2005).

De acuerdo con lo expuesto anteriormente, esta investigación analiza la incidencia del nivel de escolaridad, en las actitudes y las percepciones, frente al manejo de RAEE, provenientes de celulares y de tabletas, que tienen las personas que habitan en Florencia - Caquetá, Colombia. 


\section{MATERIALES Y MÉTODOS}

Esta investigación es de tipo descriptiva y cuantitativa, dado que la intencionalidad es observar y describir actitudes y percepciones de las personas, sin tratar de influir ni cambiar su comportamiento (Kerlinger, 2002). La población está conformada por estudiantes de primaria y secundaria de Instituciones Educativas de Florencia; estudiantes universitarios y profesionales, egresados de la Universidad de la Amazonia; esta población tiene un tamaño de 52.559 personas (DANE, 2017). Para la obtención de la población objeto de estudio, se tuvieron en cuenta los criterios de ser: a) estudiante de un grado igual o superior a segundo de cualquier Institución Educativa del área urbana de Florencia; b) estudiante de cualquier programa académico ofrecido por la Universidad de la Amazonia en Florencia y c) profesional egresado de la Universidad de la Amazonia, residente de la misma ciudad; de esta manera, el tamaño de la población objeto de estudio, se redujo a 42.913 .

Utilizando la fórmula de tamaño de muestra para obtener una proporción, con un error del $5 \%$, una confiabilidad del $95 \%$ y suponiendo una probabilidad de éxito del $50 \%$, se obtuvo, como tamaño de muestra mínimo, el de 381 personas, pero se tomó la decisión de aumentar este número a 450 personas. El muestreo, se realizó por conglomerados (para estudiantes de primaria, secundaria y universitarios), considerando, como conglomerado, la Institución Educativa. Se seleccionaron al azar 4 Instituciones Educativas y la Universidad de la Amazonia; en estas, los integrantes del semillero de investigación R3Innova de la Universidad de la Amazonia, se encargaron de aplicar las encuestas a los estudiantes seleccionados de manera aleatoria, de manera que, 152, fuesen de primaria; 149 , de secundaria y 84 , universitarios. Para los profesionales egresados de la Universidad de la Amazonia, se hizo muestreo simple aleatorio y, a los correos electrónicos registrados, se les envió la invitación de diligenciar la encuesta, junto con la URL del sitio, donde se encontraba alojada; se mantuvo disponible en la página Web, durante ocho semanas, a partir de la primera semana de agosto de 2017.

El propósito fue comprobar estadísticamente las siguientes hipótesis:

1. Cuanto más alto sea el nivel de escolaridad de la persona, sus actitudes frente al manejo de residuos electrónicos de celulares y de tabletas son más favorables con el medio ambiente.

2. Cuanto más alto sea el nivel de escolaridad de la persona, sus percepciones acerca del manejo de los residuos electrónicos provenientes de celulares y de tabletas son más favorables con el medio ambiente.

Para la aceptación o rechazo de las anteriores hipótesis, se empleó el test de independencia de variables, el cual, hace uso del estadístico $\chi^{2}$ de Pearson (Walpole \& Myers, 1992).

La encuesta, incluyó 18 preguntas, distribuidas de la siguiente manera: 5 preguntas de información general, como la edad, el género, el nivel de escolaridad y la tenencia de celular y tableta; 6 preguntas de actitudes y 7 de percepción, frente al manejo de los residuos electrónicos producidos por el desuso de celulares y de tabletas. Las preguntas enfocadas en actitudes que se tienen respecto al manejo de los residuos de celulares y de tabletas fueron planteadas para aquellas personas que hubiesen tenido o tuvieran, celular o tableta (Tabla 1).

Tabla 1. Encuesta.

\begin{tabular}{|c|c|}
\hline Pregunta & Opciones de respuesta \\
\hline Seleccione su rango de edad: & $\begin{array}{l}6 \text { a } 12 \text { años } \\
12 \text { a } 20 \text { años } \\
20 \text { a } 25 \text { años } \\
\text { Mayor de } 25 \text { años }\end{array}$ \\
\hline Género: & $\begin{array}{l}\text { Femenino } \\
\text { Masculino }\end{array}$ \\
\hline Seleccione su último grado de escolaridad: & $\begin{array}{l}\text { Primaria } \\
\text { Secundaría y/o bachillerato } \\
\text { Estudiante universitario } \\
\text { Profesional }\end{array}$ \\
\hline ¿Ha tenido o tiene celular? & $\begin{array}{l}\text { Sí } \\
\text { No }\end{array}$ \\
\hline $\begin{array}{l}\text { Razón por la que se renueva o cambia el ce- } \\
\text { lular: }\end{array}$ & $\begin{array}{l}\text { Cada vez que se daña irreparablemente } \\
\text { Cada vez que cumple su vida útil o se } \\
\text { pierde } \\
\text { Cada vez que sale un nuevo modelo }\end{array}$ \\
\hline
\end{tabular}


Continuación Tabla 1.

\begin{tabular}{|c|c|}
\hline Pregunta & Opciones de respuesta \\
\hline Si su celular deja de funcionar, usted: & $\begin{array}{l}\text { Lo manda a arreglar } \\
\text { Lo cambia }\end{array}$ \\
\hline $\begin{array}{l}\text { ¿Qué hace con las partes o con el celular } \\
\text { dañado? }\end{array}$ & $\begin{array}{l}\text { Las guarda en su casa y luego las bota } \\
\text { Las da a otra persona } \\
\text { Los lleva a un sitio de recolección } \\
\text { especializado }\end{array}$ \\
\hline ¿Ha tenido o tiene tableta? & $\begin{array}{l}\text { Sí } \\
\text { No }\end{array}$ \\
\hline $\begin{array}{l}\text { Razón por la que se renueva o cambia la tab- } \\
\text { leta: }\end{array}$ & $\begin{array}{l}\text { Cada vez que se daña irreparablemente } \\
\text { Cada vez que cumple su vida útil o se } \\
\text { pierde } \\
\text { Cada vez que sale un nuevo modelo }\end{array}$ \\
\hline Si su tableta deja de funcionar, usted: & $\begin{array}{l}\text { Lo manda a arreglar } \\
\text { Lo cambia }\end{array}$ \\
\hline $\begin{array}{l}\text { ¿Qué hace con las partes o con la tableta } \\
\text { dañada? }\end{array}$ & $\begin{array}{l}\text { Las guarda en su casa y luego las bota } \\
\text { Las da a otra persona } \\
\text { Los lleva a un sitio de recolección } \\
\text { especializado }\end{array}$ \\
\hline $\begin{array}{l}\text { ¿Sabe cuándo un dispositivo se convierte en } \\
\text { residuo electrónico? }\end{array}$ & $\begin{array}{l}\text { Sí } \\
\text { No }\end{array}$ \\
\hline $\begin{array}{l}\text { ¿Conoce sitios especializados en la } \\
\text { recolección de residuos electrónicos? }\end{array}$ & $\begin{array}{l}\text { Sí } \\
\text { No }\end{array}$ \\
\hline $\begin{array}{l}\text { ¿Cree usted que los dispositivos electrónicos } \\
\text { que no se usan o no funcionan representan } \\
\text { riesgos ambientales? }\end{array}$ & $\begin{array}{l}\text { Sí } \\
\text { No }\end{array}$ \\
\hline $\begin{array}{l}\text { ¿Cree usted que los fabricantes de dispositivos } \\
\text { electrónicos deberían diseñar dispositivos que } \\
\text { duren más? }\end{array}$ & $\begin{array}{l}\text { Sí } \\
\text { No }\end{array}$ \\
\hline $\begin{array}{l}\text { ¿Cree usted que los fabricantes de dispositivos } \\
\text { electrónicos deben proporcionar a las perso- } \\
\text { nas los medios para reciclar sus dispositivos? }\end{array}$ & $\begin{array}{l}\text { Sí } \\
\text { No }\end{array}$ \\
\hline $\begin{array}{l}\text { ¿Cree usted que los fabricantes de dispositivos } \\
\text { electrónicos deben diseñar sus dispositivos } \\
\text { de manera que se puedan reparar con más } \\
\text { facilidad? }\end{array}$ & $\begin{array}{l}\text { Sí } \\
\text { No }\end{array}$ \\
\hline $\begin{array}{l}\text { ¿Le interesaría conocer cómo cuidar nuestro } \\
\text { medio ambiente de los residuos electrónicos? }\end{array}$ & $\begin{array}{l}\text { Sí } \\
\text { No }\end{array}$ \\
\hline
\end{tabular}

Fuente: Elaboración propia.

Como software estadístico, se utilizó el SSPS Stadistics, que permitió la descripción y el análisis de la información recolectada, utilizando la $\chi^{2}$ de Pearson, como prueba de la hipótesis y de dependencia de variables.

\section{RESULTADOS Y DISCUSIÓN}

La encuesta reveló que el $24,7 \%$ de la población encuestada tenían entre 6 a 12 años; el 30,9\%, se encontraba entre 12 y 20 años; $17,1 \%$ entre 20 y 25 años y el $27,2 \%$, mayores a 25 años. El 52,4\% fue de género masculino y el $47,6 \%$, femenino. Es de anotar que de los 450 encuestados, 418 (93\% aprox.) personas afirman tener o haber tenido celular, mientras que las 32 (7\% aprox.) personas restantes manifiestan nunca haber tenido celular. Igualmente, 255 (57\% aprox.) afirman tener o haber tenido tableta y 195 (43\% aprox.) dicen nunca haber tenido tableta.

Los resultados y los análisis se presentan teniendo en cuenta las actitudes y las percepciones frente al manejo de los residuos electrónicos de celulares y de tabletas de la población analizada. 
Actitudes: Se han definido tres niveles de valoración para las actitudes: en el primer nivel, se encuentra la actitud excelente, dado que desecha el dispositivo electrónico cuando este se encuentra dañado de manera irreparable, es decir, consume responsablemente tecnología. La segunda, es la actitud aceptable, que se presenta cuando se ha cumplido el periodo de obsolescencia programado, dejando, en muchos casos, dispositivos que se encuentran en pleno funcionamiento. Finalmente, se presenta la actitud negativa, dado que adquiere y consume tecnología de manera irresponsable, obedeciendo a la satisfacción individual, sin importar las consecuencias ambientales.

Frente al cambio de celulares: De los 418 encuestados que afirman haber tenido celular, 10 tienen una excelente actitud, dado que renueva o cambia el celular cuando se daña irreparablemente; 327 tienen una actitud considerada como aceptable, relacionada con cambiar de celular cuando este cumple su vida útil o se pierde, teniendo en cuenta que, según Lezama \& Lezama (2017), la obsolescencia programa- da o vida útil de un celular es aproximadamente dos años. Finalmente, 81 tiene una actitud negativa, ya que cambian de celular cada vez que sale un nuevo modelo. Es de resaltar que prima la actitud aceptable.

Frente al cambio de tabletas: Por otra parte, de los 255 que dicen poseer o haber poseído tabletas, 6 no dieron respuesta sobre la razón de cambio de la tableta. Así, de las 249 personas que sí respondieron, 4 muestran una actitud excelente, dado que renueva o cambia la tableta cuando se daña irreparablemente; 202 tiene una actitud aceptable, relacionada con cambiar de tableta cuando esta cumple su vida útil o se pierde; finalmente, 43 tienen una actitud muy negativa, ya que cambian de tableta cada vez que sale un nuevo modelo. Es de resaltar que prima la actitud aceptable.

A pesar que el número de personas que afirman tener o haber tenido celular es casi el doble de las tabletas (418 y 255, respectivamente), el comportamiento frente a la actitud es semejante, tal como se puede apreciar en la figura $1 \mathrm{a}$.



Figura 1. Comparativo entre razones y actuaciones de propietarios de dispositivos electrónicos. 
La explicación del porqué el número de personas que afirman tener o haber tenido tableta sea menor que los que afirman haber tenido o tener celular, radica en que el consumo de tabletas está siendo desplazado por el de celulares, ya que estos últimos ofrecen las mismas características computacionales, con menor tamaño y un mayor número de funcionalidades.

Tenencia y manejo de dispositivos: Al indagar acerca de la actitud que toman, las 418 personas que afirman tener o haber tenido celular, se encontró que 292 lo manda a arreglar y 126 lo cambia; de esta manera, es mucho mayor el porcentaje de quienes tienen, a nuestro juicio, una buena actitud. En lo referente a las 255 personas que afirman tener o haber tenido tableta, 202 la mandan a arreglar y solo 53 la cambia; nuevamente, prima la actitud positiva de mandar a arreglar el dispositivo. Es de resaltar, que en lo concerniente a esta actitud tiene un mejor comportamiento los poseedores de tabletas que los de celulares, tal como se puede apreciar en la figura $1 \mathrm{~b}$.

Al preguntar a las 418 personas, que afirman tener o haber tenido celular, qué hacen con el celular o las partes que ya no les sirve, 288 indican que, lo o las guardan y, luego, lo o las botan; 108, lo o las dan a otra persona y, por lo general, la gran mayoría de estas, con el tiempo, van a parar a la basura; 22 afirman que lo o las depositan en sitios especializados de recolección (Figura 1c). Al realizar la misma pregunta a las 255 personas que afirman tener o haber tenido tableta, $56,5 \%$ mencionan que la(s) guardan y luego las botan; el $38,0 \%$ la(s) dan a otra persona (las vende o da a un técnico especializado) y, en la mayoría de los casos, terminan después de mucho tiempo, en la basura y el 5,5\% la(s) deposita(n) en un sitio de recolección especializado. En lo que respecta a esta pregunta, es bueno resaltar que la actitud que se calificó como excelente (depositar en sitio de recolección especializado), son igualmente practicadas, tanto por poseedores de celulares como los de tabletas.

Percepciones: Para estimar las percepciones de la población analizada, se consideraron siete aspectos, orientados por las preguntas relacionadas en la tabla 2 . Se evidencia, principalmente, que las personas no conocen cuándo un celular o tableta se convierte en residuo electrónico, tampoco tienen conocimiento sobre sitios especializados de recolección, para depositar este tipo de residuos, de manera responsable; más aún, son conscientes que los dispositivos no usados o no funcionales representan riesgos ambientales. Asimismo, se considera preponderante que los fabricantes de estos dispositivos, los diseñen más duraderos, de fácil reparación y proporcionen los medios para reciclar. Habría que mencionar también, la ventaja sobre el interés en cuidar el medio ambiente de los residuos electrónicos.

Dependencia de las actitudes y percepciones respecto al nivel de escolaridad: Se debe recordar que la población con nivel de escolaridad primaria es solo de 125, mientras que los de secundaria, el nivel de escolaridad, con mayor frecuencia, es de 145 y los niveles de estudiante universitario y profesional son 84 y 64 , respectivamente. Al graficar la razón por la cual se renueva o cambia el celular, por nivel de escolaridad, la peor actitud, se cambia o renueva cada vez

Tabla 2. Resultados preguntas de percepción.

\begin{tabular}{|c|l|c|c|c|c|c|}
\hline Ítem Pregunta/Percepción & \multicolumn{1}{|c|}{ Sí (\%) } & No (\%) & Valor $\chi^{2}$ & gl & Sig. \\
\hline 1 & ¿Sabe cuándo un dispositivo se convierte en residuo electrónico? & 34,7 & 65,3 & 23,092 & 3 & 0 \\
\hline 2 & $\begin{array}{l}\text { ¿Conoce sitios especializados en la recolección de residuos } \\
\text { electrónicos? }\end{array}$ & 32,7 & 67,3 & 25,212 & 3 & 0 \\
\hline 3 & $\begin{array}{l}\text { ¿Cree usted que los dispositivos electrónicos que no se usan o no } \\
\text { funcionan representan riesgos ambientales? }\end{array}$ & 82,9 & 17,1 & 33,142 & 3 & 0 \\
\hline 4 & $\begin{array}{l}\text { ¿Cree usted que los fabricantes de dispositivos electrónicos deberían } \\
\text { diseñar dispositivos que duren más? }\end{array}$ & 90,9 & 9,1 & 18,737 & 3 & 0 \\
\hline 5 & $\begin{array}{l}\text { ¿Cree usted que los fabricantes de dispositivos electrónicos deben } \\
\text { proporcionar a las personas los medios para reciclar sus dispositivos? }\end{array}$ & 94,2 & 5,8 & 28,782 & 3 & 0 \\
\hline 6 & $\begin{array}{l}\text { ¿Cree usted que los fabricantes de dispositivos electrónicos deben } \\
\text { diseñar sus dispositivos de manera que se puedan reparar con más } \\
\text { facilidad? }\end{array}$ & 94,7 & 5,3 & 3,000 & 3 & 0,392 \\
\hline 7 & $\begin{array}{l}\text { ¿́Le interesaría conocer cómo cuidar nuestro medio ambiente de los } \\
\text { residuos electrónicos? }\end{array}$ & 97,6 & 2,4 & 6,746 & 3 & 0,08 \\
\hline
\end{tabular}

Fuente: Elaboración propia. 
que sale un nuevo modelo, va decreciendo a medida que aumenta el nivel de escolaridad. Este hecho deja entrever una posible dependencia entre el nivel de escolaridad y dicha actitud. Lo anterior, no ocurre cuando se observa el comportamiento frente a qué hace si su celular deja de funcionar y la actitud que hace con las partes de su celular que ya no sirve, respecto al nivel de escolaridad.

Con el propósito de establecer la independencia entre el nivel de escolaridad y las actitudes de las personas encuestadas, en lo referente a las razones de compra de dispositivos electrónicos y en cuanto a la disposición de los residuos que resultan de esto, se realizó una prueba de independencia $\chi^{2}$, con un nivel de confianza del $95 \%$, cuyos resultados se resumen en la tabla 3.
Los niveles de significancia obtenidos en esta prueba indican que la razón por la que se renueva o cambia el celular es dependiente del nivel de escolaridad $(0,026)$. En lo que respecta a la actitud que se toma cuando su celular deja de funcionar y en lo atinente a la disposición de las partes o celular no sirven, las dos actitudes son completamente independientes del nivel de escolaridad.

En lo que respecta a las tabletas, las actitudes que tienen que ver con la razón de renovación y con la disposición de los residuos son independientes del grado de escolaridad y la única actitud que depende del grado de escolaridad es qué hace cuando el dispositivo deja de funcionar $(0,021)$.

Tabla 3. Valores del estimador Chi-cuadrado de Pearson para la prueba de hipótesis de independencia.

\begin{tabular}{|l|r|r|r|r|r|r|}
\hline \multirow{2}{*}{ Actitud } & \multicolumn{3}{c|}{ Celulares } & \multicolumn{3}{c|}{ Tabletas } \\
\cline { 2 - 7 } & Valor $\chi^{2}$ & gl & \multicolumn{1}{c|}{ Sig. } & Valor $\chi^{2}$ & \multicolumn{1}{c|}{ gl } & Sig. \\
\hline Razón por la que se renueva o cambia el dispositivo & 14,324 & 6 & 0,026 & 11,381 & 6 &, 077 \\
\hline Que hace si su dispositivo deja de funcionar & 2,503 & 3 & 0,475 & 9,726 & 3 &, 021 \\
\hline Disposición de las partes o dispositivos que ya no sirven & 5,778 & 6 & 0,449 & 2,658 & 6 &, 850 \\
\hline
\end{tabular}

Fuente: Elaboración propia.

En lo referente al análisis de la dependencia de las percepciones, respecto al nivel de escolaridad, las figuras 2 y 3 , permiten visualizar una posible dependencia de cada una, de las cinco primeras percepciones (Tabla 2), lo que se corrobora con los resultados obtenidos para $\chi^{2}$, el cual, presenta un nivel de significancia bajo. Las 2 últimas percepciones (ítem 6 y 7), son independientes del nivel de escolaridad, lo que se concluye de los resultados obtenidos en el cálculo del $\chi^{2}$ (Tabla 2).

De modo que, como factor crítico, se resalta que, una gran proporción de los encuestados, no sabe qué es un residuo electrónico, principalmente, en los niveles de primaria y de secundaria; además, en general, las personas no conocen sobre los sitios especializados de recolección. Las percepciones frente al manejo de los residuos que provienen de celulares y de tabletas dejan entrever que, principalmente, dependen del nivel de formación, además, tanto los dispositivos como sus partes, son guardados por un tiempo y, posteriormente, botados a la basura, junto con los residuos convencionales.

Un hallazgo importante consiste en la no existencia de dependencia en cuatro de las seis actitudes analizadas, respecto al nivel de escolaridad y en la existencia de dependencia para las restantes. Lo anterior permite, de manera general, afirmar que las actitudes son independientes del nivel de escolaridad, a pesar que la razón por la que se renueva o cambia el celular y el qué hace cuando su tableta deja de funcionar, son dependientes de dicho nivel.

Dado que, en seis de las siete percepciones analizadas, se evidencia una alta dependencia con el nivel de escolaridad, se deduce que dichas percepciones se encuentran influenciadas por el grado de escolaridad, de manera que, a mayor nivel de escolaridad, se tiene una mejor percepción de los riesgos potenciales de los RAEE.

Un análisis de lo encontrado en lo que respecta a las actitudes y a las percepciones es que, la forma en que actúan los habitantes de la ciudad de Florencia con respecto al manejo de los RAEE, es completamente diferente a lo que debería provocar sus percepciones. Esto demuestra dualidad de las personas, entre lo que piensan y su comportamiento.

De acuerdo con lo anterior, surgen diferentes cuestionamientos: ¿dónde se encuentra el problema?, ċos aspectos culturales inciden en el manejo inadecuado de los RAEE? cel sistema educativo considera este tipo de problemáticas ambientales? ¿cuáles son las estrategias educativas que se 

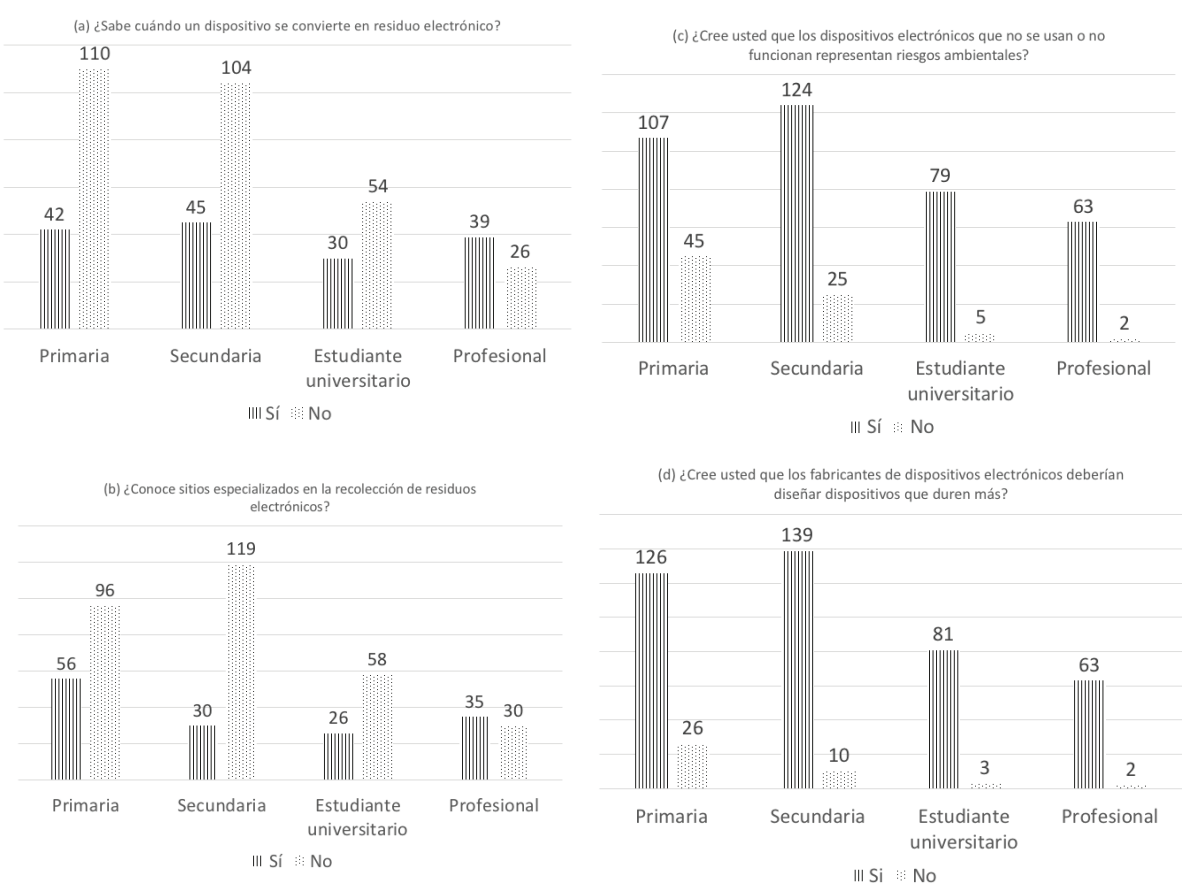

Figura 2. Percepciones de las personas encuestadas frente al manejo de los dispositivos.
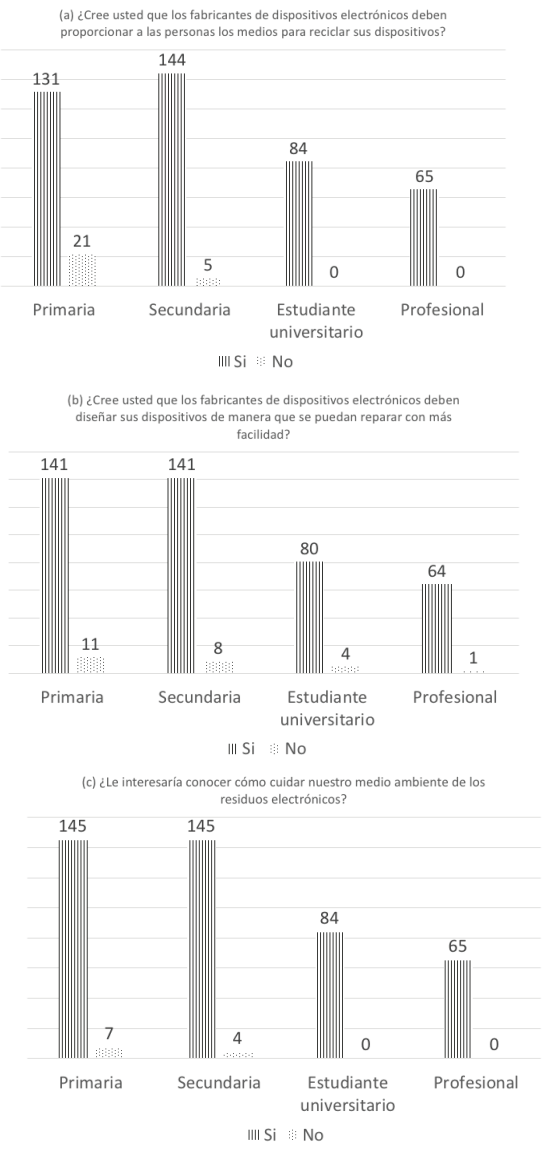

Figura 3. Percepciones de las personas encuestadas. 
deben implementar con el propósito de mejorar el comportamiento y las actitudes frente a los riesgos y manejo de los RAEE? ¿de qué manera se podría eco-alfabetizar a los niños para construir cultura ambiental?

De este estudio, se puede concluir, que no se está prestando atención a los impactos ambientales negativos, así como a los problemas de salud humana que los RAEE acarrean, debido a la falta de información, de educación y de desconocimiento sobre los riesgos de la mala manipulación de los residuos electrónicos; es irónico lo que sucede, puesto que las personas admiten y son conscientes de los problemas que acarrean, pero no dimensionan lo perjudicial que resulta para la salud, ni la afectación al medio ambiente y, por lo tanto, actúan de manera contraria.

La emergencia de la problemática, a nivel local, pone de manifiesto la necesidad de crear estrategias orientadas a que los ciudadanos mejoren sus actitudes, percepciones y conocimiento, frente al manejo de este tipo de residuos, dado que no son residuos convencionales. De ahí la importancia de trabajar desde la Amazonia, en una investigación relacionada con la cultura ambiental para el manejo de los residuos electrónicos y la innovación educativa, porque se concibe como una nueva región del conocimiento, que tiene la potencialidad de apoyar y contribuir en la solución de problemáticas ambientales, que se presentan en Colombia y el mundo.

Es así como las acciones y las estrategias que se encaminen desde la educación ambiental se deben enmarcar en los diferentes niveles de escolaridad, principalmente primaria y secundaria, direccionadas a mejorar las relaciones con el medio ambiente y fomentar el desarrollo sustentable desde el aula escolar.

La investigación presenta algunas limitaciones, que se convierten en trabajo futuro. De acuerdo con esto, se propone ahondar con técnicas cualitativas, en el análisis de las actitudes y de los comportamientos, por ejemplo, por medio de grupos focales, grupos de discusión o entrevistas, con los grupos etarios identificados con las peores actitudes, para posteriormente definir las estrategias que contribuyan a la construcción de cultura ambiental.

Conflicto de intereses: El manuscrito fue preparado y revisado con la participación de todos los autores, quienes declaramos que no existe conflicto de intereses que ponga en riesgo la validez de los resultados presentados. Financiación: Este estudio es financiado por la Universidad de la Amazonia, en el marco del Doctorado en Educación y Cultura Ambiental y el apoyo del semillero de investigación R3Innova del programa de Ingeniería de Sistemas y la Tecnología en Desarrollo de Software.

\section{BIBLIOGRAFÍA}

1. ABDOLLAHI, Y.; MOHD, S.; SAIRI, N.; ZAKARIA, A.; ABOUZARI-LOTF, E.; DORRA, M.; MOHD, M. 2015. Enhancement of electronic protection to reduce e-waste. J. Industrial and Engineering Chemistry. 29:400-407.

2. CUCCHIELLA, F.; D’ADAMO, I.; KOH, S.; ROSA, P. 2015. Recycling of WEEEs: An economic assessment of present and future e-waste streams. Renewable and Sustainable Energy Reviews. 51:263-272.

3. CHEN, J.; SHIH, L.; YANG, J.; CHEN, T. 2005. Eco-design activities in Taiwan. Environmentally Conscious Design and Inverse Manufacturing. p.535-538.

4. DANE. 2017. Investigación educación formal, matriculados zona grados escolares 2016.

5. DASKOLIA, M.; LAMBROPOULOS, N. 2009. Advancing Collaborative Creativity in the context of Greek Teachers' In-Service Training in Environmental Education. CSCLO9 Proc. $9^{\text {th }}$ international conference on Computer supported collaborative learning. 2:85-87.

6. DU BUISSON, W.; NAIDOO, R. 2014. Exploring Factors Influencing IT Workers' Green Computing Intention at a South African Firm. Proc. Southern African Institute for Computer Scientist and Information Technologists Annual Conference. ACM Press. P.148-156.

7. ESPECIALES SEMANA. 2016. La estrella fluvial de Inírida. El corazón de una selva herida y la amenaza minera. Disponible desde Internet en: http://especiales. semana.com/agua-bendita/inirida.html (con acceso 14/03/2018).

8. FISCHER, M.; HABERL, H. 2000. El metabolismo socioeconómico. Ecología Política. 19:21-34.

9. HANSELMAN, S.E.; PEGAH, M. 2007. The Wild Wild Waste: e-Waste. SIGUCCS '07 Proc. 35th annual ACM SIGUCCS fall conference. ACM Press. p.157-162.

10. JAISWAL, A.; SAMUEL, C.; PATEL, B.; KUMAR, M. 2015. Go green with WEEE: Eco-friendly approach for handling e-waste. Procedia Computer Science. 46:1317-1324.

11. KERLINGER F.; LEE, H. 2002. Investigación del comportamiento. Métodos de Investigación en Ciencias Sociales. McGrawHill (México). 850p. 
12. LEZAMA, M.; LEZAMA, E. 2017. Análisis del perfil del consumidor de celulares y sus hábitos de disposición. Strategy, Technology \& Society. 4:50-66.

13. MILOVANTSEVA, N.; FITZPATRICK, C. 2015. Barriers to electronics reuse of transboundary e-waste shipment regulations: An evaluation based on industry experiences. Resources, Conservation and Recycling. 102:170-177.

14. MINISTERIO DE AMBIENTE Y DESARROLLO SOSTENIBLE. 2018. Colombia, pionero en Suramérica en implementar políticas de gestión de RAEE. Disponible desde Internet en: http://www.minambiente. gov.co/index.php/noticias/2924-colombia-pionero-en-suramerica-en-implementar-politicas-de-gestion-de-raee (con acceso 14/05/2018).

15. ORLINS, S.; GUAN, D. 2016. China's toxic informal e-waste recycling: local approaches to a global environmental problem. J. Cleaner Production. 114:7180.

16. ÖZKIR, V.; EFENDIGIL, T.; DEMIREL, T.; DEMIREL, N.; DEVECI, M.; TOPÇU, B. 2015. A three-stage methodology for initiating an effective management system for electronic waste in Turkey. Resources, Conservation and Recycling. 96:61-70.

17. PAN, Y.; THOMAS, J. 2012. Hot or Not: A Qualitative Study on Ecological Impact of Social Media \& Fashion Consumption. Computer Supported Cooperative Work Companion. p.293-300.

18. PANAMBUNAN-FERSE, M.; BREITER, A. 2013. Assessing the side-effects of ICT development: E-waste production and management. A case study about cell phone end-of-life in Manado, Indonesia. Technology in Society. 35(3):223-231.

19. PERKINS, D.; BRUNE, M; NXELE, T.; SLY, P. 2014. E-Waste: A global hazard. Icahn School of Medicine at Mount Sinai. 80(40):286-295.

20. PICKENS, J. 2005. Attitudes and Perceptions. Organizational Behavior in Health Care. p.43-76.
21. PING, L. 2009. Strategy of green logistics and sustainable development. Information Management, Innovation Management and Industrial Engineering. 1:339342.

22. ROBINSON, B. 2009. E-waste: An assessment of global production and environmental impacts. Science Total Environment. 408(2):183-191.

23. ROKEACH, M. 1968. A theory of organization and change within value-attitudes systems. J. Sociology Issues. 24(1):13-33.

24. ROMÁN, I. 2014. eWaste en América Latina: El aporte de los operadores móviles en la reducción de la basura electrónica - Estudios de casos. Disponible desde Internet en: http://www.gsma.com/latinamerica/wp-content/uploads/2014/05/eWaste-Latam-Esp-ResEje.pdf (con acceso 27/07/2015).

25. ROY, A.; SUHONEN, J.; KIHOZA, P.; VESISENAHO, M. 2012. Promoting Education for Sustainable Development by using ICT enhanced Problem Based Learning in a developing country. Fourth International Conference on Technology for Education. 98-104.

26. SONG, Q.; LI, J. 2014. A review on human health consequences of metals exposure to e-waste in China. Environmental Pollution. 196:450-461.

27. TORRES, J. 2015. Nuevos desiertos avanzan detrás de la fiebre del oro. El Tiempo. Disponible desde Internet en: http://www.eltiempo.com/archivo/documento/ CMS-16460299

28. WALPOLE, R.; MYERS, R. 1992. Probabilidad y estadística. McGrawHill (México). 736p.

29. ZHANG, X.; LONG LUO, N.; SUN, C. 2009. The Knowledge building of the Eco-Classroom. Sixth International Conference on Information Technology: New Generations. p.1100-1103.

Recibido: Marzo 13 de2018

Aceptado: Mayo 31 de 2018

Cómo citar:

Pascuas R., Y.; Chico V., D.; Hernández R., S. 2018. residuos de celulares y tabletas: incidencia del nivel de escolaridad en las actitudes y percepciones frente a su manejo.Rev. U.D.C.A Act. Div. Cient.21(1): 243-252. 